\title{
P 248 IMPLEMENTATION OF A PILOT MULTIDISCIPLINARY TEAM MEETING FOR SUPPORTIVE CARE IN ADVANCED LUNG DISEASE ( SCALD MDTM)
}

Laura McTague, ${ }^{1}$ Mudher Al-khairalla, ${ }^{2}$ Ruth McCook, ${ }^{3}$ Debbie Edwards, ${ }^{3}$ Dawn Warner ${ }^{3} .{ }^{1}$ St Johns Hospice, Doncaster, United Kingdom; ${ }^{2}$ Doncaster and Bassetlaw NHS Foundation Trust, United Kingdom; ${ }^{3}$ Rotherham, Doncaster and South Humber Mental Health Care Trust

10.1136/bmjspcare-2014-000654.289

Background Locally, only $12 \%$ of patients with advanced lung disease die at home. There is a significant rate of readmission to hospital in the last year of life. Most patients are housebound. Contact with Specialist Consultants and advance care planning is ad hoc.

The idea to develop the Multidisciplinary team meeting (MDTM) arose from the Acute Hospital Senior Clinicians EOLC development project and a local review of COPD services. The pilot took place within current local resources.

Aim The Supportive Care in Advanced Lung Disease (SCALD) MDTM aimed to support clinical staff in identifying the last year of life. The MDTM would review appropriate investigations, discuss and communicate advance care planning and optimal symptom control to all involved.

Method The MDTM included a respiratory consultant, specialist palliative care consultant and community respiratory nurse specialists. Patients gave verbal consent to the community respiratory nurses or referrer for the MDTM discussion to take place. The meeting was held at monthly intervals. A template was designed to record the MDTM and a letter was sent out the same day by the MDTM lead. The referrer returned to the patient to discuss the outcomes.

Results A survey of 20 patients was carried out. The majority of patients had severe COPD, were housebound and on long term oxygen therapy. Advance care planning including plans for appropriate ceilings of care and preferred place of death were improved as a result of the MDTM. Other outcomes included specialist palliative care consultant home visits and respiratory consultant clinic review.

Discussion The SCALD MDTM design and implementation resulted in improved advance care planning and Consultant contact. A larger pilot and research would give increased knowledge about the impact of this service design on the experience of advanced lung disease patients in the last year of life. 